\title{
The effect of rotating the distal arm in the Poggendorff configuration: A replication
}

\author{
A. W. PRESSEY \\ University of Manitoba, Winnipeg, Canada R3T 2N2
}

\begin{abstract}
Weintraub and Krantz (1971) rotated the distal oblique line in the Poggendorff configuration and found that distortion decreased at first and then increased as angle increased. The present study used a similar target to the one employed by Weintraub and Krantz but a different psychophysical method. The previous results were verified. It was argued that the distal contours do play a role in the Poggendorff configuration and that theories which focus exclusively on the acute angle must be reevaluated.
\end{abstract}

In the Poggendorff illusion, shown in Figure 1A, two portions of a single oblique line do not appear colinear if they are interrupted by two parallel lines:

The most popular explanation of the Poggendorff illusion is that distortion occurs because acute angles are perceptually enlarged. Thus, in Figure 1, the oblique line on the left turns away from the vertical line and appears to point above the oblique line on the right. The cause of such enlargement of acute angles has been attributed to sensory blurring (Chiang, 1968), spurious excitation (Walker, 1972), distorted sensory input (Burns \& Pritchard, 1971), and lateral inhibition between orientation detectors (Blakemore, Carpenter, $\&$ Georgeson, 1970). The greatest difficulty that these theories face is the repeated finding that removing all the contours except those that form acute angles results in a reduction or a reversal of the Poggendorff effect (Day, 1973; Green \& Hoyle, 1964; Judd, 1899; Pressey \& den Heyer, 1968; Restle, 1969).

Another problem for theories that focus exclusively on the role of the acute angle is that manipulation of the distal line alters the illusion. Thus, Pressey and Sweeney (1972) found that the amount of illusion as measured by angular distortion increased as the distance between parallel lines increased. Such an effect is not predictable from a theory which claims that the sole cause of the Poggendorff illusion is the presence of an acute angle.

Another effect which is consequent upon a variation in the distal contour was reported by Weintraub and Krantz (1971). They measured the illusion by adjusting the lower left oblique line; the independent variable consisted of rotating the distal oblique line from 0 to $129.8 \mathrm{deg}$ (see Figure 1B). Their results showed that, as angle $\mathrm{A}$ increased, the illusion decreased at first and then increased, until at $129.8 \mathrm{deg}$ it was larger than at $0 \mathrm{deg}$. However, there is a possibility that their data are not reliable, since they did not provide a statistical evaluation.

Because the results of Weintraub and Krantz (1971)

This study was supported by the National Research Council of Canada (Grant AO-177). Reprints may be obtained from the Department of Psychology, University of Manitoba, Winnipeg, Manitoba R3T 2N2.

provide one of the few pieces of evidence that the distal contours in the Poggendorff target affect the magnitude of distortion, we wished to verify these findings with a different psychophysical method.

\section{METHOD}

\section{Subjects}

The subjects were 40 men and women enrolled in introductory psychology at the University of Manitoba. Only individuals who had good vision, with or without glasses, were asked to participate.

\section{Apparatus}

The apparatus consisted of a white target holder that was $36.8 \mathrm{~cm}$ wide and $39.5 \mathrm{~cm}$ high. The face of the holder was tilted backwards 20 deg in order to insure that the entire target was perpendicular to the line of sight. A chinrest was placed in front of the holder so that the distance between the subject's eyes and the face of the frame was approximately $41 \mathrm{~cm}$.

The targets were drawn with India ink on sheets of white paper, each of which was $278 \mathrm{~mm}$ high and $214 \mathrm{~mm}$ wide. The basic configuration consisted of two vertical lines which were $278 \mathrm{~mm}$ long and $30 \mathrm{~mm}$ apart. The constant oblique line joined the vertical line at a distance of approximately $85 \mathrm{~mm}$ from the top or bottom edge of the sheet, and the variable (or rotated) oblique line joined the opposite vertical line also at a distance of $85 \mathrm{~mm}$ from the edge of the sheet. The constant oblique line formed an angle of 16 deg with the proximal vertical line, and the rotated oblique line formed an angle of 0 (no oblique line), $8,31,50,67,90$, and $130 \mathrm{deg}$ with the other (distal) vertical line. Each oblique line was $60 \mathrm{~mm}$ long. A target which contained a dot at the point of objective continuation on the distal vertical line was also employed.

The nine targets were presented in one of four orientations defined by the direction in which the constant oblique line pointed. The directions were up-right; up-left; down-right; and down-left. (The direction shown in Figure 1B is up-right.)

A
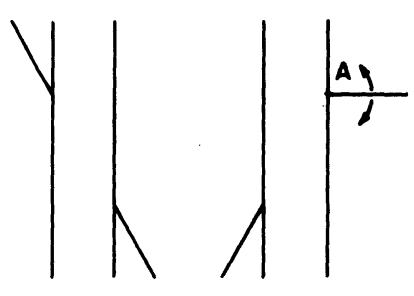

Figure 1. The Poggendorff configuration (A) and the configuration with a rotated distal oblique line (B). 


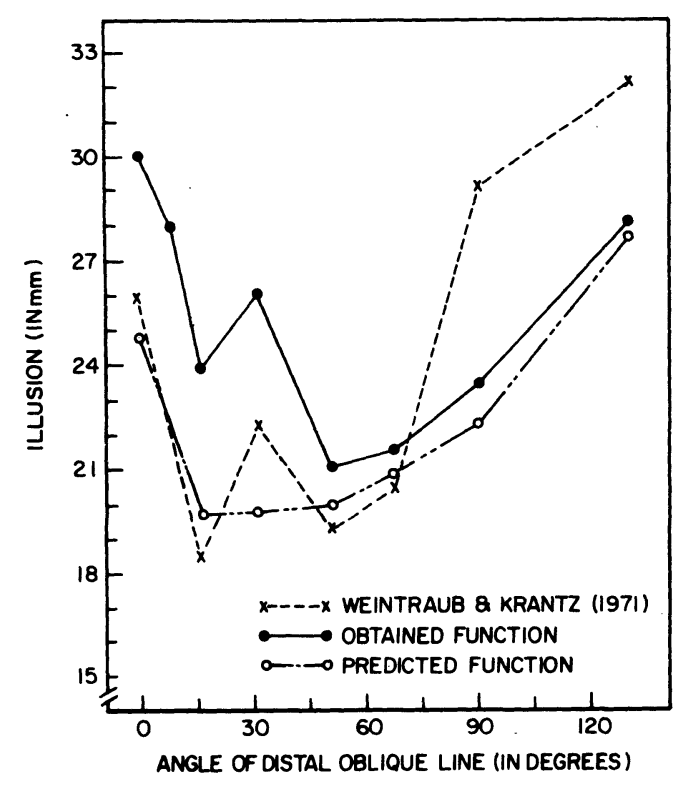

Figure 2. The Poggendorff illusion as a function of angle of distal oblique line. The predicted function is from Pressey and Sweeney (1972).

All targets were reproduced by offset duplicating procedures.

\section{Procedure}

A split-plot design was employed in which the angle of the distal arm was a within-subjects variable and orientation was a between-subjects variable. There were an equal number of subjects in each group. In addition, four successive replications were carried out. Within each replication the order of presenting targets was randomized for each subject.

Subjects were tested individually by an experimenter who was not familiar with the expected findings. Each subject was seated in front of the apparatus, placed his chin on the rest, and was shown a sample target formally similar to the experimental targets. Subjects were asked to extend visually the constant oblique line until it crossed the opposite vertical line and to mark the point of crossing with a small but distinct dot. No mention was made of the dot or the oblique line on the distal vertical line, but, if subjects asked, they were told not to pay any particular attention to them. The targets were presented manually and subjects were allowed as much time as necessary to respond.

\section{RESULTS}

Measurements were made with a millimeter scale and were accurate to within $.5 \mathrm{~mm}$. The score was defined in relation to the objective continuation of the constant oblique line; responses in the direction of the classical illusion were scored as positive and in the opposite direction as negative. A preliminary analysis of the data showed that whatever effect was there tended to be eliminated in the later replications, and thus only the first replication was employed. The main effect of rotation of the distal oblique line is shown in Figure 2. Also shown in Figure 2 are the data from
Weintraub and Krantz (1971) and the predicted function from Pressey and Sweeney (1972). Clearly, the U function reported by Weintraub and Krantz is a reliable one, especially since the method of measuring the illusion was so different in the present study.

Analysis of variance showed that only the main effect of angle of the rotated oblique line was statistically significant $(\mathrm{F}=3.60, \mathrm{df}=7,252, \mathrm{p}<.05)$. Thus, whether the standard oblique line was on the left or right side or whether it pointed up or down seemed to have little effect on the illusion.

A target in which a dot was located on the distal line (at the point of objective continuation of the standard oblique line) was included in order to employ a condition used by Weintraub and Krantz. The mean illusion on this target was $25.36 \mathrm{~mm}$, which was significantly smaller than the $30.03 \mathrm{~mm}$ of illusion found when no dot was employed $(\mathrm{t}=2.51, \mathrm{df}=39, \mathrm{p}<.05)$. It is not possible to say, at this time, whether the dot reduces the amount of distortion per se or whether it simply acts as an anchor to change the response. Nevertheless, the larger illusion which results from eliminating the distal dot seems consistent with the finding that eliminating both obliques as objective stimuli tends to enhance distortion (Pressey \& Wilson, 1974).

\section{DISCUSSION}

The fact that rotation of the distal line alters the magnitude of distortion indicates that the theories which focus exclusively on the acute or obtuse angle formed by the proximal arm are not sufficient to explain the Poggendorff effect. On the other hand, these data enhance the validity of the assimilation theory of the Poggendorff illusion (Pressey, 1971), which argues that it is the distal contour that is of primary importance. Of course, these results do not bear on the question of whether the Poggendorff effect can be explained solely on the basis of the distal contours. There is some evidence that it cannot be so explained. For example, Weintraub and Krantz retained only the proximal contour and a dot at the distal extension of the standard oblique line. A substantial Poggendorff effect still occurred. It seems, therefore, that both the proximal and the distal contours play a role, but the exact manner in which those contours mediate the Poggendorff illusion remains to be specified.

\section{REFERENCES}

Blakemore, C., Carpenter, R. H., \& Georgeson, M. A. Lateral inhibition between orientation detectors in the human visual system. Nature, 1970, 228, 37-39.

Burns, D. B., \& Pritchard, $R$. Geometrical illusions and the responses of neurones in the cat's visual cortex to angle patterns. Journal of Physiology, 1971, 213, 599-616.

Chiang, C. A new theory to explain geometrical illusions produced by crossing lines. Perception \& Psychophysics, 1968 , 3, 174-176.

Day, R. H. The Poggendorff illusion with acute and obtuse angles. Perception \& Psychophysics, 1973, 14, 590-596.

Green, R. T., \& Hoyle, E. M. The influence of spatial orientation on the Poggendorff illusion. Acta Psychologica, 1964, 22, 348-366.

Judd, C. H. A study of geometrical illusions. Psychological Review, 1899, 6, 241-261.

Pressey, A. W. An extension of assimilation theory to illusions of size, area, and direction. Perception \& Psychophysics, $1971,9,172-176$. 
Pressey, A. W., \& den Heyer, K. Observations on Chiang's "new" theory of geometrical illusions. Perception \& Psychophysics, $1968,4,313-314$.

Pressey, A. W. \& Sweeney, O. Some puzzling results on the Poggendorff illusion. Perception \& Psychophysics, 1971, 9, $172-176$.

Pressey, A. W., \& Wilson, A. E. The Poggendorff illusion in imagination. Bulletin of the Psychonomic Society, 1974, 3, 447-449.

Restle, F. Illusions of bent line. Perception \& Psychophysics, $1969,5,273-274$.
Walker, E. H. A mathematical theory of optical illusions and figural aftereffects. Perception \& Psychophysics, 1973 $13,467-486$

Weintraub, D. J., \& Krantz, D. The Poggendorff illusion: Amputations, rotations, and other perturbations. Perception \& Psychophysics, 1971, 10, 257-264.

(Received for publication March 4, 1976.) 